\title{
Akupunktur bei Sportverletzungen
}

\author{
Weniger Schmerzen und raschere Regeneration -
} Die Akupunktur bildet bei Sportverletzungen eine wirksame und nebenwirkungsarme Option

Sven Weisser

Sportunfälle, sei es im Vereins- oder Freizeitsport, sind häufige Verletzungen. Es wird geschätzt, dass sich pro Jahr ca. 1,3 Mio. Bundesbürger beim Sport so schwer verletzen, dass sie ärztlich behandelt werden müssen [2]. Die jährlichen Kosten belaufen sich nach Schätzungen auf ca. 1,650 Mio.€ (Stand 2000). Bei den Männern treten oft Verletzungen bei den großen Ballsportarten (Fußball, Handball, Volleyball, Basketball) auf, bei den Frauen neben Hand- und Volleyball auch bei Gymnastik und Turnen. Die am meisten verletzte Körperregion ist das Sprunggelenk, gefolgt vom Kniegelenk und der Schulter.

Die Akupunktur hat sich in der Sportmedizin bei Verletzungen als wichtiger Bestandteil der Therapie etabliert. Bei korrekter Anwendung können die Schmerzen gelindert sowie eine rasche Regeneration gefördert werden - ohne wesentliche Nebenwirkungen.

Die Akupunktur bietet Behandlungsmöglichkeiten bei knöchernen sowie bei Weichteilverletzungen. Der Beitrag gibt einen Überblick zu den Möglichkeiten der Akupunktur in der konservativen Therapie von Verletzungsfolgen.

\section{Konservative Therapie von} Verletzungen mit Akupunktur Die Akupunktur kann bei folgenden Verletzungen des Bewegungsapparats sinnvoll eingesetzt werden:

\section{Akute Verletzungen}

- Verletzungen der Bänder

- Muskelverletzungen wie Muskelfaserrisse, Muskelreizungen und Verhärtungen - Schmerztherapie
Chronische Verletzungen

- Tennisellenbogen / Golferellenbogen

- Schmerzhafte Schultersteife (Frozen Shoulder)

- Kniebeschwerden

- Achillessehnenentzündungen

- Sehnenscheidenentzündungen

- Patellaspitzen-Syndrom (Jumpers Knee)

- Nacken- und Rückenschmerzen

- Schleimbeutelentzündungen

Postoperative Situationen, z. B.:

- Förderung der Wundheilung

- Vorbeugen von Entzündungen

- abschwellende, schmerzlindernde,

lymphflussanregende Wirkung

\section{Allgemeine Hinweise}

Nach einem Sportunfall wird der Patient zuerst nach den Kriterien der Schulmedizin

\section{Zusammenfassung}

Die Akupunktur hat sich in der Sportmedizin bei akuten und chronischen Verletzungen als wichtiger Therapiebestandteil etabliert. Besonders beim Schmerzmanagement und zur Verkürzung der Regenerationszeit bietet sie eine nebenwirkungsarme Option. Der Beitrag stellt die Möglichkeiten der Akupunktur bei häufigen Weichteilverletzungen im Sport vor. 
Bei Sportverletzungen hat sich der Einsatz von Mikroakupunktursystemen bewährt und wird mit der Körperakupunktur kombiniert. In der Praxis wird am häufigsten die Ohrakupunktur sowie die Neue Schädelakupunktur nach Yamamoto (YNSA) angewendet.

Bei der Ohrakupunktur ist die obere Extremität im Bereich der Scapha, die untere Extremität in der Fossa triangularis lokalisiert. Irritable Punkte werden detektiert und akupunktiert. Weitere irritierte Punkte werden durch den vektoriellen Behandlungsstrahl nach Nogier gefunden und ebenfalls akupunktiert. Dabei wird der durch die Palpation empfindlichste Ohrpunkt gedanklich mit dem Nullpunkt verbunden und diese Linie sorgfältig weiter auf empfindliche Punkte untersucht.

Weiterhin werden häufig die sich auf der Rückseite der Ohrmuschel befindlichen „Retro-Punkte“ verwendet, da sie eine hohe Effektivität bei motorischen Funktionsstörungen aufweisen. Die „Retro-Punkte“ haben nahezu die gleiche Indikation wie die entsprechenden Punkte auf der Ohrvorderseite.

Bei der Neuen Schädelakupunktur nach Yamamoto repräsentieren die BasisPunkte/Zone Teile des Bewegungssystems. Die A-Zone repräsentiert den Kopf/Nacken, die B-Zone den HWS-Nacken-Schulter-Bereich, die C-Zone in den „Geheimratswinkeln“ die obere Extremität, die D-Zone die LWS und untere Extremität. Bei einer exakten Nadelung der empfindlichen Punkte kommt es häufig zu einer sofortigen Beschwerdelinderung.

untersucht. Dazu gehört neben der körperlichen Untersuchung in den meisten Fällen eine radiologische Bildgebung (konventionelles Röntgen) zum Ausschluss einer knöchernen Verletzung. Die im Folgenden aufgeführten Akupunkturpunkte haben sich in der Praxis bei den jeweiligen Indikationen bewährt. Jeder Patient ist nach den Regeln der Traditionellen Chinesischen Medizin zu untersuchen, um die entsprechenden Akupunkturpunkte auszuwählen.

Adjuvant zur Akupunktur ist eine Physiotherapie zu empfehlen. Sollte sich im Rahmen der TCM-Diagnostik eine Kältesymptomatik zeigen, kann Moxibustion ergänzend angewendet werden. Bei einer Winderkrankung hat sich die Schröpfkopftherapie als günstig erwiesen. Zeigt sich bei der klinischen Untersuchung eine Blutstase, kann blutig geschröpft werden.

\section{Sprunggelenk}

Die häufigste Sprunggelenksverletzung beim Sport ist die Distorsion, ggf. mit einer Läsion der Außenbänder. Nach Ausschluss einer Außenknöchelfraktur sollte unverzüglich mit der Akupunktur begonnen werden. Bei einer Hauptbeschwerdesymptomatik im Bereich des Lig. fibulotalare anterius werden Punkte im Verlauf des Gallenblasenmeridians, bei Schmerzen im Bereich des Lig. fibulotalare posterius oder des Lig. fibulocalcaneare Punkte im Blasenmeridian akupunktiert.

Bei der Sprunggelenksdistorsion zeigt sich in den meisten Fällen eine lokale QiStagnation mit Blutstase. Häufig liegt eine muskuläre Dysbalance der Unterschenkelmuskulatur vor.

\section{Akutphase}

In der Akutphase ist es nicht angebracht, in die geschwollenen Weichteile des betroffenen Sprunggelenks zu akupunktieren. Es werden v.a. Punkte auf der kontralateralen Seite akupunktiert. Je nach Schmerzlokalisation sind dies meist

- bei einer Außenbandsymptomatik: Bl60, Bl62, evtl. Bl61, Bl63 und Gb40,

- bei einer Symptomatik im Innenknöchelbereich: Ma41, Ni3, Ni6, Mi5.

Auf der betroffenen Seite kann im Akutfall lokal eine Lasertherapie durchgeführt werden.

\section{Bei anhaltenden Beschwerden}

Nach Abklingen der akuten Symptomatik mit Schwellung und Hämatom kann bei anhaltender Beschwerdesymptomatik auch das betroffene Sprunggelenk direkt akupunktiert werden. Nach Distorsionen bilden sich häufig Triggerpunkte, deshalb sollte zusätzlich die Peronaeusgruppe auf Triggerpunkte untersucht werden.

Bei aktiven Triggerpunkten empfiehlt sich das „dry needling“ dieser Punkte. Bei chronischen Beschwerden hat sich nach Ausschluss von Kontraindikationen die Moxibustion der betroffenen Akupunkturpunkte als hilfreich erwiesen.

In einer 2013 veröffentlichten Studie konnte nachgewiesen werden, dass die Propriozeption nach einer Distorsion des oberen Sprunggelenks durch Akupunktur mit Moxibusion signifikant verbessert werden konnte. 

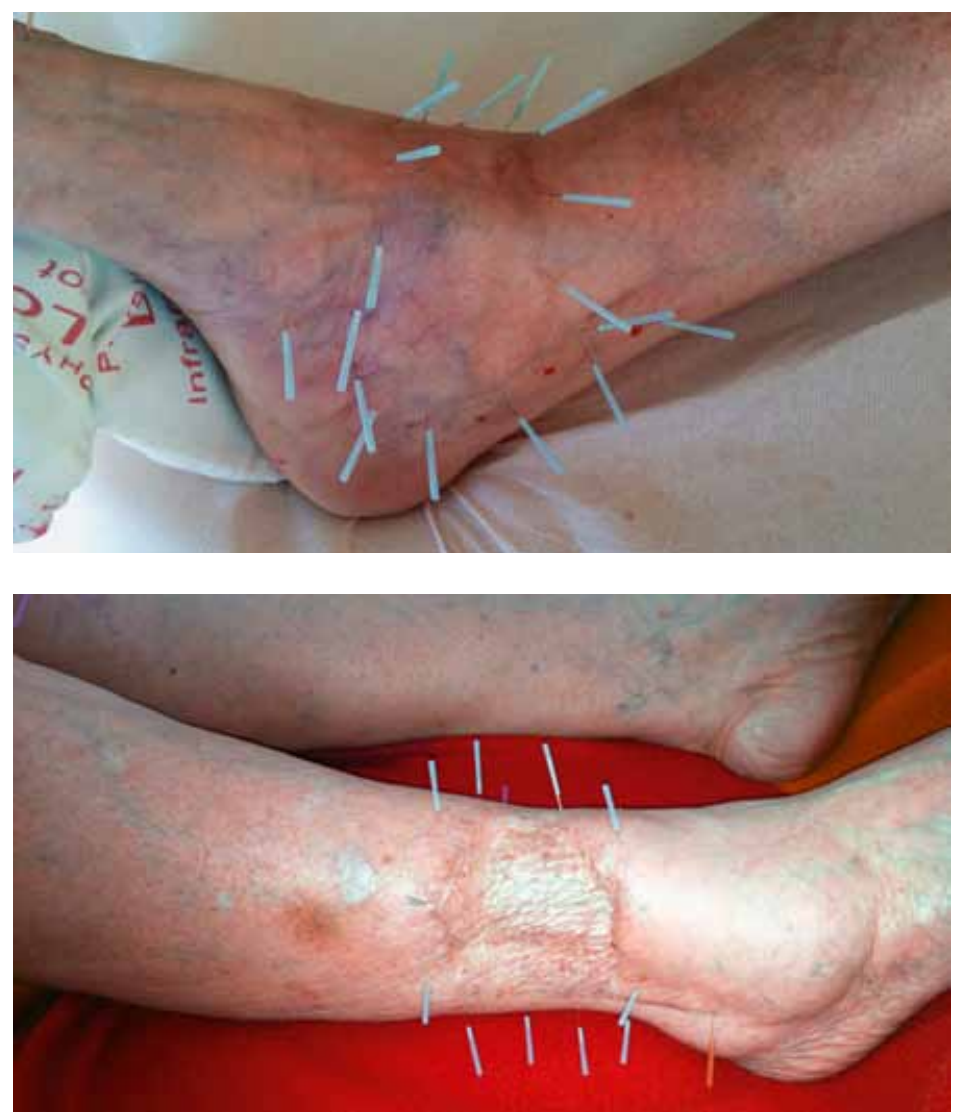

Abb. 2 Chronischer Schmerz nach tiefer Weichteilverletzung mit Gewebedefekt und Hauttransplantation - Behandlung mit lokaler Nadelung. (c) Wolfram Stör

Die Kontrollgruppe wurde mit Physiotherapie behandelt. Die genadelten Punkte waren Gb40, Bl60, Bl62, Ma41, und Ashi-Punkte [3].

\section{Achillessehne}

Die schulmedizinische Therapie bei chronischen Achillessehnenbeschwerden ist uneinheitlich und es gibt viele Therapieansätze ohne durchschlagende Erfolge. Hier kann die Akupunktur ebenfalls gute Dienste leisten.

\section{Akutphase}

Im akuten Stadium ist das Nadeln im entzündeten Gewebe kontraindiziert, vielmehr ist die kontralaterale Seite heranzuziehen.

\section{Chronische Beschwerden}

Im nicht akuten Stadium können lokale Punkte wie $\mathrm{Bl} 60$ und $\mathrm{Ni} 3$ genadelt werden. Bei chronischen Achillessehnenbeschwerden hat es sich bewährt, den muskulären Status der Unterschenkelmuskultur zu evaluieren. Sehr häufig finden sich Triggerpunkte direkt im M. soleus und M. gastrocnemius oder in der anatagonistischen Muskulatur des M. tibialis anterior. Aktive Punkte sind oft bei Bl57 und Ma 36 zu finden, sodass es sich anbietet, diese Punkte bei Achillodynien mit zu nadeln.

\section{Kniegelenk}

Neben den Sprunggelenksverletzungen spielen akute Verletzungen des Kniegelenks in der Sportmedizin eine große Rolle. Auch hier kann auf eine ausführliche schulmedizinische Untersuchung nicht verzichtet werden, um eine Kniebinnenverletzung wie Verletzung der Seitenbänder, der Kreuzbänder, der Menisken, der Patellar-/Quadrizepssehne sowie Frakturen auszuschließen.

\section{Cave: Generell sollte bei allen Akupunk- turbehandlungen im Kniegelenksbereich darauf geachtet werden, dass keine Nadel intraartikulär zu liegen kommt.}

\section{Akute Kniegelenkschmerzen}

Bei akuten Kniegelenkschmerzen nach einer Distorsion oder Prellung werden ähnlich wie bei der Sprungelenksdistorsion im Akutstadium Fernpunkte auf dem betroffenen Meridian (in Höhe des Sprunggelenks oder des Ellenbogengelenks) bzw. Punkte im Bereich des kontralateralen Kniegelenks genadelt. Nach Abklingen der lokalen Weichteilschwellung wird auch am betroffenen Kniegelenk direkt akupunktiert. Entscheidend hierbei ist auch die Palpation des umgebenden Gewebes, da sich häufig schmerzhafte Punkte finden, die keinem Meridian zugeordnet werden können (sog. Ashi-Punkte). Diese Punkte werden dann ebenfalls wie Nahpunkte akupunktiert.

\section{Chronische Kniegelenkschmerzen}

Von 2002-2007 wurden in Deutschland die groß angelegten German Acupuncture Trials durchgeführt [1]. In den Studien standen u.a. die therapeutischen Erfolge bei Gonar- throse mit der Anwendung von Akupunktur im Vergleich zu Schein-Akupunktur und konservativer Standardtherapie im Fokus. Bei der Schein-Akupunktur wurden z.T. auch Punkte im Segment genadelt, d.h. die Akupunktur erfolgte an nicht klassischen Punkten. Eingeschlossen wurden 1007 Gonarthrosepatienten, die mind. 6 Monate über Kniegelenksbeschwerden klagten. Die in den Studienarm „Akupunktur“ oder „Schein-Akupunktur“ eingeschlossenen Patienten erhielten bis zu 10 Akupunktursitzungen innerhalb von 6 Wochen.

Nach einem halben Jahr wurden die Patienten bez. Schmerz und Funktion (nach dem WOMAC-Score) befragt. Dabei zeigte sich sowohl die Akupunktur (Erfolgsrate $53 \%$ ) als auch die Scheinakupunktur (Erfolgsrate $51 \%$ ) der konservativen Therapie (Erfolgsrate 29,1\%) deutlich überlegen.

In einem systematischen Review konnten White et al. nachweisen, dass eine signifikante Überlegenheit von Akupunktur im Vergleich zur Sham-Akupunktur in Bezug auf Schmerz und Beweglichkeit bei chronischem Knieschmerz vorliegt. Auch in der Langzeitbeobachtung (52 Wochen nach Studienbeginn) war die Überlegenheit gegenüber der Sham-Akupunktur noch signifikant [4]. Interessanterweise ist die Studienlage für Akupunktur bei vielen Erkrankungen besser als für konventionelle Therapien. So gibt es bislang keine Studie, die den Nutzen einer langfristigen NSAR-Therapie bei chronischen Knieschmerzen untersucht hat.

Bei chronischen Kniegelenkschmerzen werden in der Akupunktur sowohl Nahpunkte als auch Fernpunkte eingesetzt:

Bei retropatellarer Schmerzsymptomatik sowie bei muskulären Dysbalancen im M. vastus medialis werden häufig Ma 35, EX-UE 4, Mi 10 und Ma 34 eingesetzt.

Bei Insertionstendinopatie der Quadrizeps-Muskulatur kann die sog. „PatellaSpinne“ genadelt werden, d. h. es werden ca. 6-10 Nadeln lokal um die Patella gesetzt.

Liegt eine ödematöse Schwellung vor, empfiehlt es sich, Punkte der Milz-Leitbahn mit zu nadeln.

Auch vor der geplanten Implantation einer Kniegelenks-Totalendoprothese empfiehlt es sich, die muskuläre Dysbalance durch eine verkürzte dorsale Oberschenkelmuskulatur (die meisten Patienten mit einer Gonarthrose weisen eine Streckhemmung im Kniegelenk auf) durch Akupunktur zu verbessern. 


\section{Rückenschmerzen}

Im Rahmen von Sportverletzungen treten häufig Rückenschmerzen auf. Abzugrenzen ist der akut aufgetretene Rückenschmerz von den chronischen Lumbalgien. Aus Sicht der TCM ist der akute Rückenschmerz häufig als eine einschießende Winderkrankung einzuordnen, ggf. kombiniert mit einer Kälte- oder Feuchtigkeitssymptomatik. Anatomisch-funktionell liegt hierbei meistens eine Funktionsstörung der Muskulatur und der Facettengelenke vor.

Je nach Art des Traumas (Sturz, direktes Anpralltrauma) muss eine radiologische Bildgebung zum Ausschluss einer Fraktur durchgeführt werden. Nach Ausschluss einer strukturellen Wirbelsäulenverletzung kann unverzüglich mit der Akupunktur begonnen werden.

Im Rahmen von myogenen Funktionsstörungen werden neben den Fernpunkten lokale druckdolente Akupunkturpunkte gestochen. Bei lokaler Fülle und bei Blutstase bietet sich hier auch blutiges Schröpfen an. Dabei werden kleinere Hautverletzungen durch eine Nadel gesetzt und darüber der Schröpfkopf platziert. Durch den Blutaustritt kommt es zu einer Ableitung der lokalen Füllesymptomatik.

Fernpunkte werden ableitend behandelt. So hat sich das blutige Nadeln von Bl 40 (in der Kniekehle lokalisiert) als sehr hilfreich erwiesen. Man kann gezielt durch kleinere Venen akupunktieren und so einen Mikroaderlass hervorrufen: z.B. kann Bl 40 mit einer dünnen Kanüle (G 22) gestochen werden.

Als weitere Fernpunkte werden Akupunkturpunkte der Shao-Yang-Leitbahn bei akuter vivinderkrankung akupunktiert: Gb 34, Le 3, 3E5 und LG 20 kommen häufig zum Einsatz. Als hilfreiche Fernpunkte sind darüber hinaus Dü 3 und Bl 62 als Einschaltpunkte von außerordentlichen Meridianen (Lenkergefäß und Yang Qiao Mai) nicht zu vergessen.

Bei akuten Rückenschmerzen nach Verletzungen können arthrogene Funktionsstörungen zugrunde liegen. Dabei kommt es zu Segmentblockierungen zwischen den Wirbelkörpern. In Vorbereitung auf eine manualtherapeutische Intervention kann die Akupunktur adjuvant eingesetzt werden, da jene mit muskulären Verspannungen einhergehen.

Bei einer neurogenen Schmerzsymptomatik sollte Akupunktur nur nach Abklärung der Ursache eingesetzt werden. Neurologische Symptome sollten bildgebend durch MRT weiter abgeklärt werden.

Bei akuter radikulärer Symptomatik mit starker Schmerzhaftigkeit und Hyperästhesie sollte nicht lokal akupunktiert werden. Hier bieten sich die o.g. Fernpunkte an, besonders Bl 40, 60, 62, evtl. Bl 67.

\section{Schultergelenk}

Erkrankungen des Schultergelenkes sind häufig und vielfältig. So können akute Verletzungen wie Distorsionen, Rotatorenmanschettenrupturen oder Verletzungsfolgen wie Bursitiden auftreten, aber auch chronische Verletzungen wie Impingementsyndrom, Ansatztendinosen, Tendinitien, Kapselstörungen wie Frozen Shoulder oder Arthrosen.

Aus Sicht der TCM kommt es durch Trauma, Überlastung oder äußere pathogene Faktoren v.a. zu einer Störung des Qi- und BlutFlusses. Die Lokalisation des Schulterschmerzes (ventral, lateral oder dorsal) ist für die Wahl der Akupunkturpunkte von entscheidender Bedeutung. Je nach Lokalisation sind unterschiedliche Achsen (Yang Ming, Shao Yang, Tai Yang) betroffen und die jeweils entsprechenden Akupunkturpunkte zu wählen.

\section{Ventraler Schulterschmerz}

Bei einem ventralen Schulterschmerz sind v.a. die Yang-Ming- (Di/Ma) und die Tai-YinAchse (Lu/Mi) betroffen.

Als häufigste Fernpunkte werden Lu5, Lu 7, Di 4, Di 10, Ma 38 und Mi 9 genadelt. Eine herausragende Wirksamkeit bei Schulterschmerzen weist Ma 38 auf. Wichtig ist, den Punkt kräftig zu stimulieren und den Patienten aufzufordern, die Schulter während der Stimulation zu bewegen.

Als lokale Punkte werden Punkte wie Di 14, Di 15 eingesetzt.

\section{Lateraler Schulterschmerz}

Bei einem lateralen Schulterschmerz ist die Achse Shao Yang (3E/Gb) betroffen: Hier werden sehr oft Punkte wie 3E4, 3E5 oder Gb 34 ausgewählt. 


\section{Dorsaler Schulterschmerz}

Wenn der Schulterschmerz dorsalseitig lokalisiert wird, werden vorzugsweise Punkte der Tai Yang Achse (Dü/Bl) ausgewählt. Die lokalen Punkten wie Dü 9, Dü 10, Dü 11, Dü 12, Dü 13 werden mit den Fernpunkten der betroffenen Achse (Dü 3, Dü 6, Bl39, Bl40, Bl60) kombiniert. Bei der klinischen Untersuchung ist auf schmerzhafte Verspannungen wie Myogelosen und Triggerpunkte der gesamten Schultermuskulatur zu achten. So können u.a. Triggerpunkte im M. infraspinatus und M. supraspinatus einen ventralen Schulterschmerz hervorrufen und sollten mitbehandelt werden

\section{Tennisellenbogen}

Bei der Epicondylopathia humeri beschreiben die Patienten einen Schmerz im Ellenbogenbereich bei Strecken des Handgelenks und der Langfinger. Ätiologisch liegt häufig eine chronische mechanische Überbeanspruchung im Ansatzbereich der Unterarmmuskulatur vor, die Streckmuskulatur ist davon weitaus häufiger betroffen als die Beugemuskulatur. Eine Epikondylitis humeri kann ebenfalls nach dem Ausüben einer ungewohnten Tätigkeit auftreten. Klinisch sind ein Druckschmerz im muskulären Ansatzbereich sowie eine Schmerzauslösung beim Anspannen der Unterarmmuskulatur gegen einen Widerstand zu finden.

Im Sinne der TCM liegt meist eine Qi-Stagnation, häufig kombiniert mit einer Blutstagnation, vor. In der klinischen Untersuchung zeigen sich oft Triggerpunkte in der Unterarmmuskulatur sowie Ansatztendinosen bei häufig verkürzter Muskulatur.

Bei einer Epikondylitis radialis (Tennisellenbogen) ist das Augenmerk auf den M. extensor carpi radialis, den M. extensor digitorum, den M. supinatus und den M. brachioradialis zu richten.

Bei einer Epikondylitis ulnaris sind v.a. die Flexorengruppe der Hand und der Finger sowie der M. pronator teres betroffen.

Irnich et al. konnten in einer kontrollierten Studie zeigen, dass die Patienten schon nach der ersten Akupunkturbehandlung eine deutliche Linderung der Beschwerden vorweisen konnten. Folgende Akupunkturpunkte wurden genadelt: Di4, Di10, Dü3, 3E5 und Gb34 [5].

\section{Ein Fall aus der Praxis}

32 Jahre alter Handballspieler. Nach einem Handballspiel Schmerzen in der rechten
Schulter. Ein eindeutiges Trauma während des Spieles nicht erinnerlich.

Schmerzlokalisation: Schulter ventralseitig. Erstvorstellung beim Orthopäden: konventionelle Röntgendiagnostik ohne pathologischen Befund. Einleitung der Therapie mit NSAR. Darunter nur kurze Zeit Besserung der Beschwerden. Aufgrund der anhaltenden Beschwerden wurde ein MRT des rechten Schultergelenks durchgeführt. Dabei zeigte sich eine intakte Rotatorenmanschette, ein intaktes Labrum, eine unauffällige Bizepssehne. Pathologisch zeigte sich eine leichte Bursitis subacromialis. Beginn mit Physiotherapie. Bei anhaltenden Beschwerden wurde die diagnostische Arthroskopie des rechten Schultergelenks empfohlen. Aus beruflichen Gründen wünschte der Patient vorerst keine operative Versorgung, sondern weiterhin die konservative Therapie. Aus diesem Grund erfolgte die Vorstellung zur Akupunktur.

Bei der Diagnostik nach den Regeln der TCM zeigte sich eine sog. Außenerkrankung mit lokaler Füllesymptomatik. Aufgrund der ventralen Schmerzlokalisation war die Leitbahn Yang-Ming betroffen.

Akupunkturbehandlung: Es wurde mit der Akupunktur von Punkten auf der Lungen-/Dickdarmleitbahn begonnen und zusätzlich Ma 38 mitgenadelt und kräftig stimuliert. Nach 3 Behandlungen im Abstand von 4 Tagen zeigte sich eine deutliche Beschwerdebesserung mit längeren schmerzfreien Intervallen. Der Patient kann nachts wieder durchschlafen. Bei noch nicht vollständiger Schmerzfreiheit wurde die Rotatorenmanschettenmuskulatur nochmals untersucht. Im Befund zeigten sich aktive Triggerpunkte im M. infraspinatus, die auf Druck den ventralen Schulterschmerz auslösten. In der Folge wurden nun zweimalige „Triggerpunkt-Akupunktursitzungen“ mit dryneedling der Triggerpunkte im Infraund Supraspinatus durchgeführt. Danach hatte der Patient so gut wie keine Beschwerden mehr in der rechten Schulter.

\section{Fazit}

Mit der Akupunktur hat man ein Werkzeug zur Hand, das Patienten mit Sportverletzungen eine zusätzliche wirksame Behandlungsoption eröffnet. Ein Kochbuch für die adjuvante Therapie mit Akupunktur bei Verletzungsfolgen kann der Artikel jedoch nicht bieten, entscheidend für eine erfolgreiche Behandlung sind die Anamnese nach den Regeln der TCM und die anschließende für jeden Patienten individuelle Punktauswahl.

Interessenkonflikt: Der Autor erklärt, dass keine wirtschaftlichen oder persönlichen Verbindungen bestehen.

\section{Online zu finden unter}

http://dx.doi.org/10.1055/s-0034-1390669

\section{Literatur}

1 Scharf HP, Mansmann U, Streitberger K et al. GERAC. Acupuncture and knee osteoarthritis: a three-armed randomized trial. Ann Intern Med 2006; 145 (1): 12-20

$\overline{2}$ Gläser H, Henke T. Sportunfälle - Häufigkeit, Kosten, Prävention. Hrsg. von der ARAG Allgemeine Versicherungs-AG in Zusammenarbeit mit der Ruhr-Universität Bochum, Lehrstuhl für Sportmedizin. Düsseldorf 2002

3 Zhu Y, Qiu ML, Ding Y, et al. Effects of electroacupuncture on the proprioception of athletes with functional ankle instability. [Article in Chinese]. Zhongguo Zhen Jiu 2012; 32 (6): 503-506

4 Vas J, White A. Evidence from RCTs on optimal acupuncture treatment for knee osteoarthritis - an exploratory review. Acupunct Med 2007; 25 (1-2): 29-35

5 Irnich D, Karg H, Behrens N, et al. Controlled trial on point specificity of acupuncture in the treatment of lateral epicondylitis (tennis elbow). Phys Med Rehab Kur Med 2003; 13 (4): 215-219

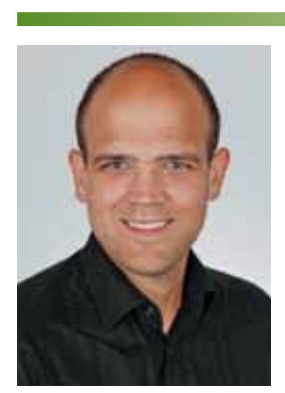

Dr. Sven Weisser

Facharzt für Orthopädie und Unfallchirurgie Berufsgenossenschaftliche Unfallklinik Tübingen Schnarrenbergstr. 95 72076 Tübingen sweisser@bgu-tuebingen.de

Sven Weisser begann bereits während des Medizinstudiums mit dem Studium der Akupunktur. 2004 DÄGfA-A-Diplom und Tutor bei der DÄGfA; 2006 Approbation als Arzt; 2009 Zusatzbezeichnung Akupunktur; 2013 Facharzt für Orthopädie und Unfallchirurgie 